\title{
Neutron streaming studies along JET shielding penetrations
}

\author{
Ion E. Stamatelatos ${ }^{1,}$ a , Theodora Vasilopoulou ${ }^{1}$, Paola Batistoni $^{2}$, Barbara Obryk $^{3}$, \\ Sergey Popovichev ${ }^{4}$, Jonathan Naish ${ }^{4}$, and JET contributors* \\ EUROfusion Consortium, JET, Culham Science Centre, OX14 3DB, Abingdon, United Kingdom \\ ${ }^{1}$ Institute of Nuclear and Radiological Sciences, Technology, Energy \& Safety, NCSR "Demokritos", Athens, Greece
2 ENEA - Fusion Technical Unit, Frascati, Rome, Italy
${ }^{3}$ Institute of Nuclear Physics, Polish Academy of Sciences, Kraków, Poland
${ }^{4}$ CCFE, Culham Science Centre, OX14 3DB, Abingdon, United Kingdom
${ }^{*}$ See the appendix of Romanelli F. et al, 2014, Proc. 25th IAEA Fusion Energy Conference 2014, St. Petersburg, Russia
}

\begin{abstract}
Neutronic benchmark experiments are carried out at JET aiming to assess the neutronic codes and data used in ITER analysis. Among other activities, experiments are performed in order to validate neutron streaming simulations along long penetrations in the JET shielding configuration. In this work, neutron streaming calculations along the JET personnel entrance maze are presented. Simulations were performed using the MCNP code for Deuterium-Deuterium and DeuteriumTritium plasma sources. The results of the simulations were compared against experimental data obtained using thermoluminescence detectors and activation foils.
\end{abstract}

\section{Introduction}

The Joint European Torus (JET) is currently the largest tokamak in the world. The experiments and design studies performed by JET are consolidated to a large extent into the design of its successor ITER. Among others, benchmark experiments are being carried out at JET aiming to validate in a real fusion environment the neutronic codes and nuclear data applied in ITER nuclear analyses. In particular, measurements and calculations of the neutron fluence through the penetrations of the JET shielding aim to assess the capability of numerical tools to accurately predict neutron transport along the long paths and the complex geometries characterizing the ITER biological shield [1].

In the present work, neutron streaming through the JET Hall South West personnel entrance maze was evaluated. Monte Carlo calculations using the MCNP code were performed to predict neutron fluence and ambient dose equivalent along the maze. DeuteriumDeuterium (D-D) and Deuterium-Tritium (D-T) toroidal plasma sources were simulated. The results of the calculations were validated by comparison against measurements carried out using thermoluminescence detectors and activation foils.

\section{Simulations}

The personnel entrance maze is located at the South West (SW) corner of the JET Hall (Fig.1). The geometry and dimensions of the maze are shown in Figure 2.

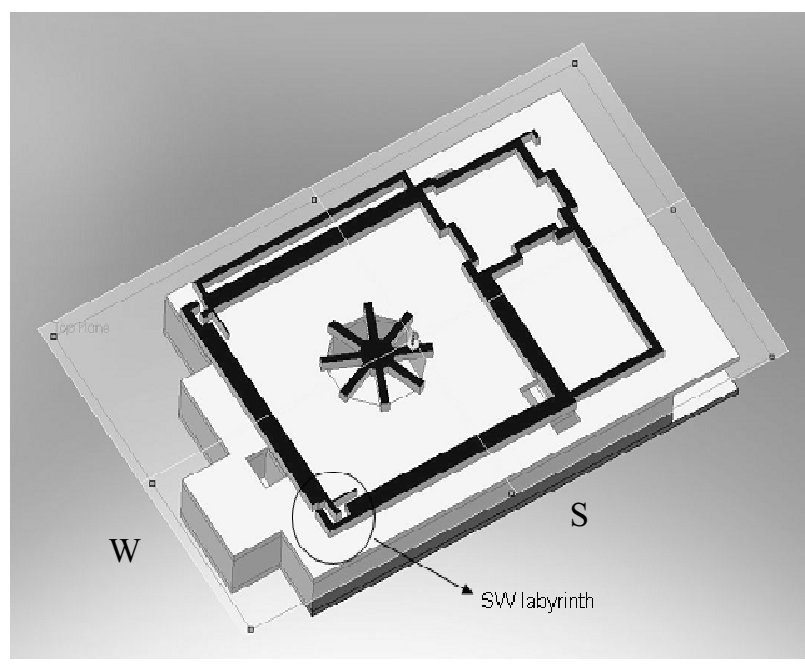

Figure 1. Ground view of JET Hall showing the location of the SW personnel entrance maze

\footnotetext{
${ }^{\mathrm{a}}$ Corresponding author: ion@ipta.demokritos.gr
} 


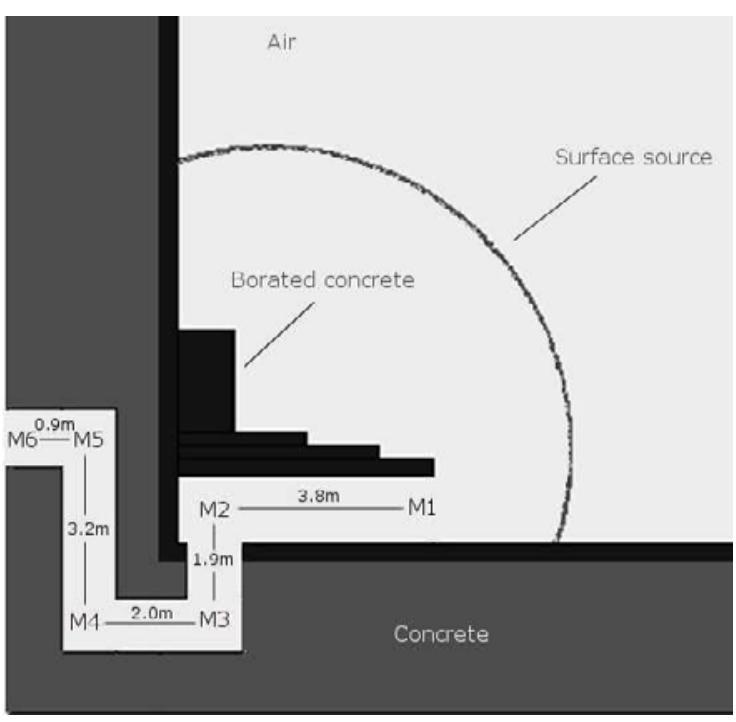

Figure 2. Cross sectional view of the SW entrance maze

The maze configuration provides four right-angle turns. The total length of the maze is $11.80 \mathrm{~m}$ and its height is $2.60 \mathrm{~m}$. The maze width varies between 0.90 and $1.10 \mathrm{~m}$ and therefore the cross-sectional area ranges between $2.34 \mathrm{~m}^{2}$ and $2.86 \mathrm{~m}^{2}$. The thickness of the concrete wall is $2.50 \mathrm{~m}$. The internal surface of the wall is covered by a layer of borated concrete $(0.30 \mathrm{~m}$ in thickness $)$. The densities of concrete and borated concrete were $2.43 \times 10^{3}$ $\mathrm{kg} \cdot \mathrm{m}^{-3}$ and $2.20 \times 10^{3} \mathrm{~kg} \cdot \mathrm{m}^{-3}$, respectively.

Simulations were performed for D-D and D-T toroidal plasma sources using Monte Carlo code MCNPX (version 2.5.0) [2]. Cross-section data were obtained from JEFF 3.1.2 and FENDL 2.1 libraries [3, 4]. All calculations were performed for the "as built" concrete compositions. The detailed concrete composition has been given elsewhere [5].

A two-stage simulation approach was employed. A detailed model of the JET Torus was used to produce a Surface Source Write file registering the neutrons directed towards the SW corner of the JET Hall. The model took into consideration the actual toroidal distribution of the neutrons produced in the JET plasma. The modeled geometry included the plasma facing components, the vacuum vessel, the magnetic coils, the shell, transformer limbs and concrete shielding walls. Outside the shell and before the walls, however, there are numerous substantial structures which have not been modeled in detail (diagnostic and heating systems, various equipments). Moreover, the vacuum vessel has ports through which neutrons may escape. These complications were approximated with a zone composed of iron, plastic and copper which has been adjusted in thickness until the JET external fission chamber measurements were consistent with the fluxes in the MCNP model [1]. This approximation was necessary since the complexity in the torus hall is too great for more detailed modeling on a realistic timescale. The Surface Source Write file registered neutrons on a spherical surface with center at the SW Hall corner $(1.0 \mathrm{~m}$ above the floor level) and radius of $5.0 \mathrm{~m}$ (Fig. 2). The Surface Source Write file was used as Surface Source Read input file for the subsequent maze calculations performed in the context of this work.

Neutron fluence was calculated using track length estimate tallies of neutron flux in spherical cells of $0.3 \mathrm{~m}$ in radius positioned along the maze at $1.0 \mathrm{~m}$ height from the floor level. Moreover, ambient dose equivalent, $\mathrm{H}^{*}(10)$, was calculated folding neutron flux by ambient dose equivalent to neutron fluence conversion factors as a function of neutron energy [6]. It is noted that the ambient dose equivalent $\mathrm{H}^{*}(10)$ at a point of interest in a radiation field is the dose equivalent which would be generated in the associated oriented and expanded radiation field at a depth of $10 \mathrm{~mm}$ on the radius of the ICRU sphere which is oriented opposite to the direction of incident radiation. Statistical uncertainties were kept below $10 \%$ for all track length estimate tallies.

\section{Results}

Figures $3 \& 4$ show the MCNP predicted neutron energy spectrum at positions M1-M6 along the labyrinth for D-D and D-T JET plasma sources, respectively. Position M1 corresponds to the inner entrance of the maze (mouth) and position M6 to the exit door.

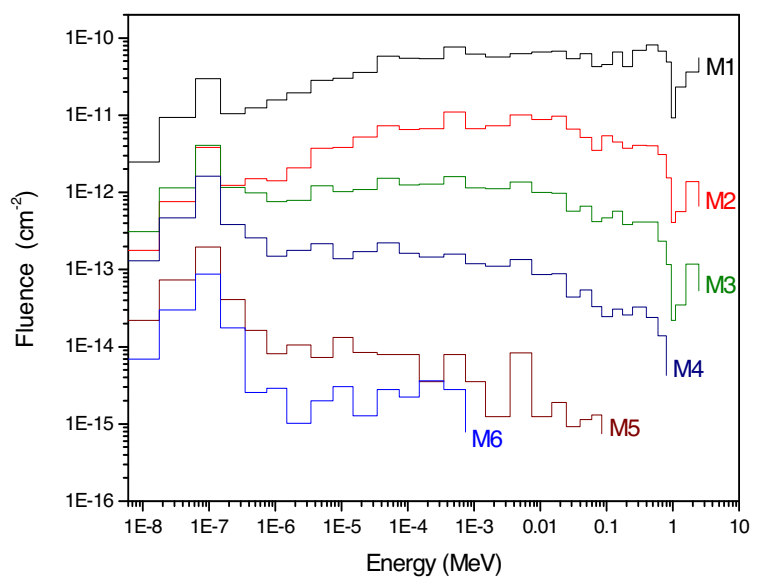

Figure 3. Neutron energy spectrum for the D-D source

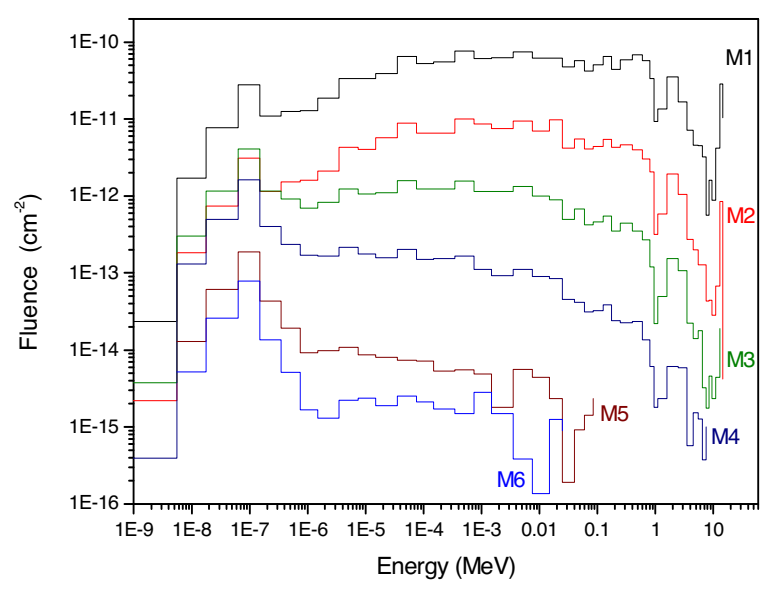

Figure 4. Neutron energy spectrum for the D-T source 
The neutrons that are directed towards the labyrinth mouth have already been scattered by the machine, surrounding structures and wall materials one or more times and have lost a significant fraction of their kinetic energy. The mean energy of neutrons at the labyrinth mouth (M1) for the D-D and D-T source was approximately $270 \mathrm{keV}$ and $650 \mathrm{keV}$, respectively. However, during their propagation along the maze, neutrons are elastically scattered by the wall materials and are further slowed down. The mean neutron energy at labyrinth position M3 for the D-D and the D-T source was $40 \mathrm{keV}$ and $70 \mathrm{keV}$, respectively. At the exit (M6) neutron energy was in the $\mathrm{eV}$ region for both sources.

Figures 5 \& 6 show the MCNP calculated neutron fluence along the maze, as a function of the summed centerline distance (L) from the inner maze entrance (mouth) to the exit door, divided by the square root of the maze cross-sectional area (A) for the D-D and D-T plasma sources, respectively. The results were normalized per JET source neutron. In these figures neutron fluence is presented in three energy groups: "thermal" $(\mathrm{E}<0.5$ $\mathrm{eV})$, "epithermal" $(0.5 \mathrm{eV}<\mathrm{E}<0.1 \mathrm{MeV})$ and "fast" $(\mathrm{E}>0.1 \mathrm{MeV}$ ). The total neutron fluence (sum of the three groups) is also shown.

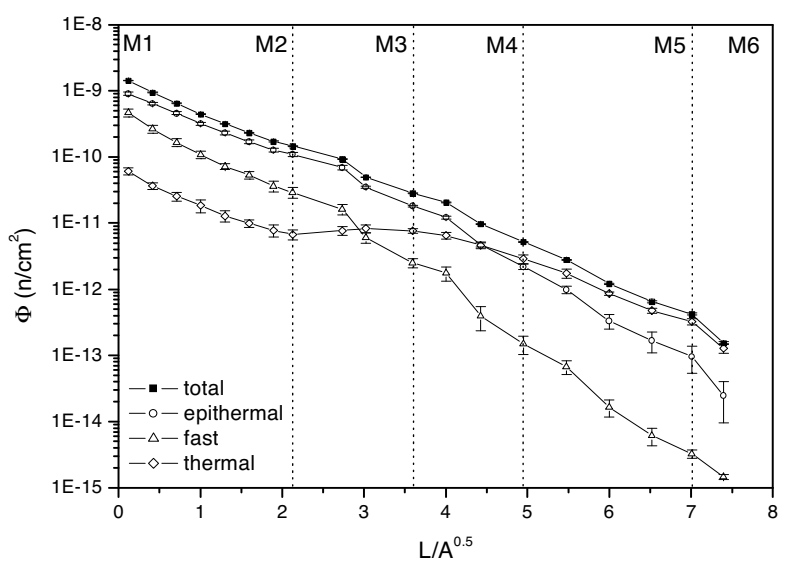

Figure 5. Neutron fluence for the D-D plasma source (normalization per JET neutron)

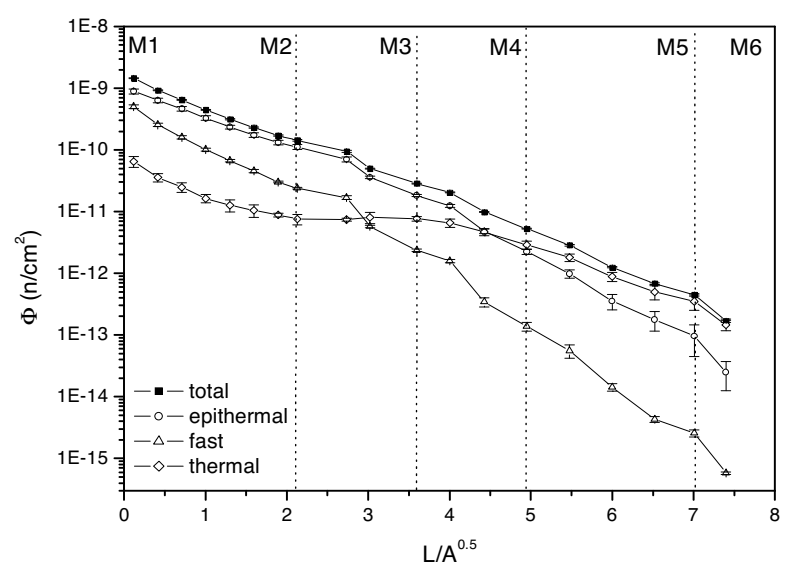

Figure 6. Neutron fluence for the D-T plasma source (normalization per JET neutron)
From Fig. 5 it can be seen that the total neutron fluence is attenuated along the maze (total length of 11.8 $\mathrm{m})$ by about four orders of magnitude. The neutron fluence at the labyrinth exit was $(1.7 \pm 0.1) \times 10^{-13} \mathrm{~cm}^{-2}$ per JET neutron. In the first two legs of the labyrinth (sections M1-M2 and M2-M3) neutron fluence is dominated by epithermal neutrons. However, for $\mathrm{L} / \mathrm{A}^{0.5} \geq$ 4.5 the thermal neutron group becomes the dominant one. At the labyrinth mouth (M1) the relative contribution of the fast, epithermal and thermal groups to the total neutron fluence was found to be $34 \%, 62 \%$ and $4 \%$, respectively. At the second right-angle bend of the labyrinth (M3) the relative contribution to neutron fluence of the fast, epithermal and thermal groups was found to be $9 \%, 64 \%$ and $27 \%$, respectively. At the exit door (M6), the relative contribution of fast, epithermal and thermal groups was $1 \%, 15 \%$ and $84 \%$, respectively. Since the neutron fluence at the labyrinth exit is dominated by low energy neutrons, a further attenuation of the neutron fluence can easily be achieved by using a thermal neutron absorbing material (i.e. ${ }^{6}$ Li-doped polyethylene) at the door shield. A similar behavior was also observed for the D-T plasma source (Fig. 6).

Figure 7 shows the MCNP calculated ambient dose equivalent, $\mathrm{H}^{*}(10)$, along the maze as a function of the parameter $\mathrm{L} / \mathrm{A}^{0.5}$ for the $\mathrm{D}-\mathrm{D}$ and $\mathrm{D}-\mathrm{T}$ sources. The results are normalized per JET neutron.

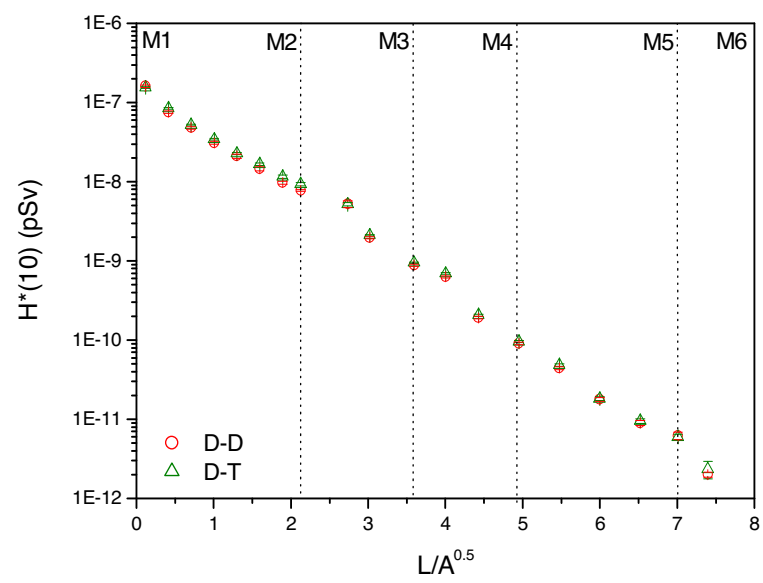

Figure 7. Neutron ambient dose equivalent, $H^{*}(10)$, as a function of $\mathrm{L} / \mathrm{A}^{0.5}$ for $\mathrm{D}$-D and $\mathrm{D}$-T plasma sources (per JET neutron)

As it can be seen from Fig. 7, in both source cases the ambient dose equivalent is decreasing along the total length of the maze. The $H^{*}(10)$ at the maze exit for the $\mathrm{D}-\mathrm{D}$ and the D-T source was of $(2.0 \pm 0.2) \times 10^{-12} \mathrm{pSv}$ and $(2.3 \pm 0.6) \times 10^{-12} \mathrm{pSv}$ per JET neutron, respectively. The maze transmission factor was found to be $1.2 \times 10^{-5}$ and $1.5 \times 10^{-5}$ for the D-D and the D-T source, respectively. The maze transmission factor was defined as the ratio of the calculated ambient dose equivalent at the maze exit (M6) over the calculated dose at the maze mouth (M1) and is considered to be an index of the effectiveness of the maze in attenuating neutrons. The comparable calculated $\mathrm{H}^{*}(10)$ values at the maze exit in D-D and D-T operation modes (per JET neutron) are 
attributed to the fact that neutrons entering the maze have already been slowed down due to multiple interactions in the Torus and wall materials and their propagation along the maze has become independent of their initial energy at the point of production (source). It is stressed that the results shown in Fig. 7 depend on the maze geometric configuration and shielding materials employed. Therefore, they cannot be readily generalized to other neutron sources and maze configurations as well.

\section{Validation}

A benchmark experiment was performed in the 20152016 JET D-D campaign in order to validate the results of the calculations. Measurements were performed using MCP-type thermoluminescence detectors (TLDs) and activation foils. The TLDs were produced and measured by the Institute of Nuclear Physics, Polish Academy of Sciences, Krakow, Poland. TLDs were positioned in rectangular and circular holders within large polyethylene cylindrical moderators $(\mathrm{d}=25 \mathrm{~cm}, \mathrm{H}=25 \mathrm{~cm})$ [7]. The activation foils used were Tantalum foil discs (bare and Cadmium covered). The foils were measured by the NCSR "Demokritos" group. The detectors were positioned at selected locations in the JET Hall and shielding penetrations (Fig. 8).

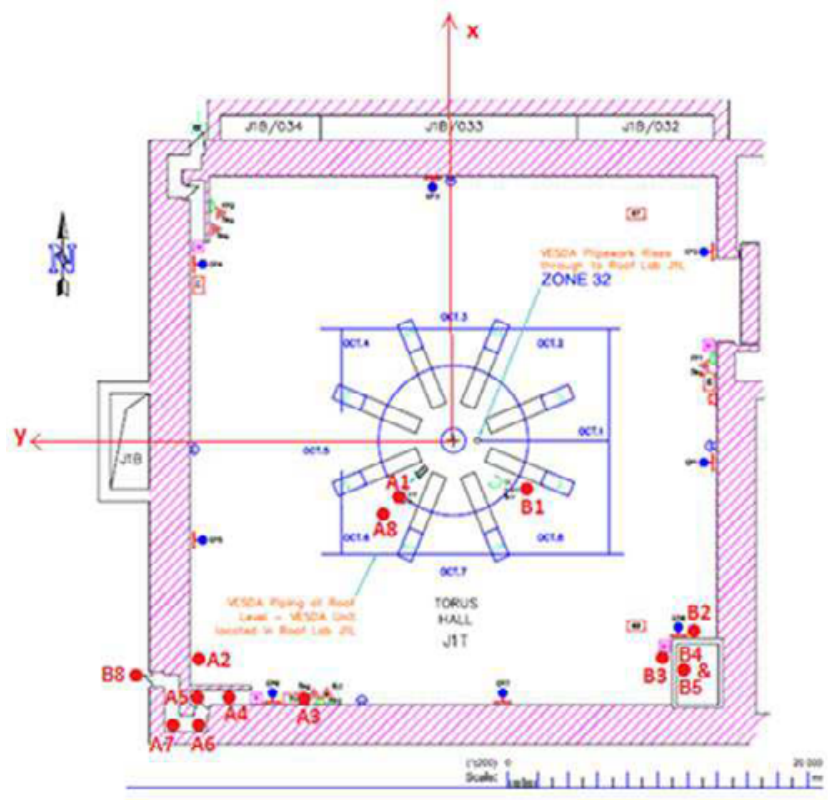

Figure 8. Detector locations in the Torus hall

To produce results comparable to those derived from the TLDs, the activation foils were positioned within large polyethylene moderator cylinders at selected locations within the JET hall, close to the moderators containing the TLDs (Fig. 9). More details on the experimental procedure have been discussed elsewhere [1].

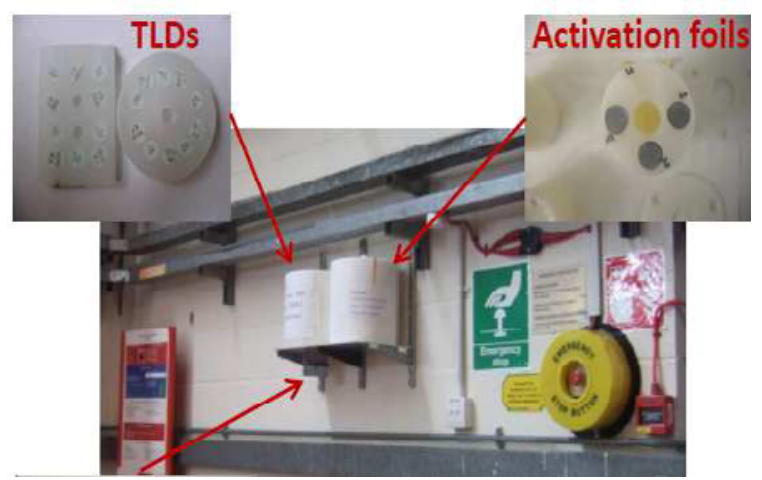

Figure 9. Detector positioning for irradiation in the Hall

The comparison of the simulations against the experimental measurements is presented in Table 1 . In this table, the neutron fluence results derived using Tantalum foils and TLDs as well as the MCNP calculated values are given along with their $\%$ relative errors for five detector positions. One of these detectors (A1) was located near the tokamak, two detectors (A2 \& A4) in the SW labyrinth region and two more detectors (B3 \& B5) in the South East corner near the chimney (Fig. 8).

The neutron fluence values shown in Table 1 correspond to a total budget of $3.52 \mathrm{E}+18$ neutrons, which is the total amount of neutrons produced at the source during the experimental campaign.

It is stressed that attention was paid in the development of the MCNP model and the subsequent calculations in order to ensure that the results will be comparable to those derived using the TLDs. For this reason, all associated parameters (such as the neutron self-shielding effect as well as the "shadowing" effect caused by the interference between individual TLDs and foils within the moderator) have been studied and taken into account in the calculations. A detailed description of the method has been presented $[1,7]$.

Table 1. Neutron fluence results for selected detector positions at the JET Hall

\begin{tabular}{|c|c|c|c|c|c|}
\hline \multirow{3}{*}{$\begin{array}{l}\text { Detector } \\
\text { Position }\end{array}$} & \multicolumn{5}{|c|}{$\begin{array}{l}\text { Neutron fluence }\left(\mathrm{cm}^{-2}\right) \\
(\% \text { rel.error })\end{array}$} \\
\hline & \multirow{2}{*}{ Ta foil } & \multicolumn{2}{|c|}{ TLDs } & \multicolumn{2}{|c|}{$\begin{array}{l}\text { MCNPX } \\
\text { calculations }\end{array}$} \\
\hline & & Circle & Square & Circle & Square \\
\hline $\mathrm{A} 1$ & $\begin{array}{l}2.7 \mathrm{E}+10 \\
(16.4)\end{array}$ & $\begin{array}{c}2.5 \mathrm{E}+10 \\
(7.3)\end{array}$ & $\begin{array}{l}2.7 \mathrm{E}+10 \\
(6.0)\end{array}$ & - & - \\
\hline $\mathrm{A} 2$ & $\begin{array}{l}1.0 \mathrm{E}+9 \\
(20.0)\end{array}$ & $\begin{array}{c}1.2 \mathrm{E}+9 \\
(7.0)\end{array}$ & $\begin{array}{c}1.3 \mathrm{E}+9 \\
(6.6)\end{array}$ & $\begin{array}{c}2.2 \mathrm{E}+9 \\
(5.2)\end{array}$ & $\begin{array}{c}2.3 \mathrm{E}+9 \\
(5.2)\end{array}$ \\
\hline A4 & $\begin{array}{l}2.2 \mathrm{E}+8 \\
(33.2)\end{array}$ & $\begin{array}{c}3.9 \mathrm{E}+8 \\
(6.6)\end{array}$ & $\begin{array}{c}5.1 \mathrm{E}+8 \\
(7.8)\end{array}$ & $\begin{array}{c}1.4 \mathrm{E}+9 \\
(4.1)\end{array}$ & $\begin{array}{c}1.4 \mathrm{E}+9 \\
(4.1)\end{array}$ \\
\hline B3 & $\begin{array}{l}9.6 \mathrm{E}+8 \\
(18.9)\end{array}$ & $\begin{array}{l}9.5 \mathrm{E}+8 \\
(7.3)\end{array}$ & $\begin{array}{c}1.0 \mathrm{E}+9 \\
(6.3)\end{array}$ & $\begin{array}{c}1.6 \mathrm{E}+9 \\
(5.4)\end{array}$ & $\begin{array}{c}1.7 \mathrm{E}+9 \\
(5.4)\end{array}$ \\
\hline B5 & $\begin{array}{c}2.1 \mathrm{E}+8 \\
(43.3)\end{array}$ & $\begin{array}{c}3.4 \mathrm{E}+8 \\
(6.9)\end{array}$ & $\begin{array}{c}4.0 \mathrm{E}+8 \\
(7.2)\end{array}$ & $\begin{array}{c}1.6 \mathrm{E}+8 \\
(7.1)\end{array}$ & $\begin{array}{c}1.7 \mathrm{E}+8 \\
(7.1)\end{array}$ \\
\hline
\end{tabular}


As it can be seen from Table 1, a good agreement was observed between TLDs and activation foil measurements for most of the positions studied. At detector positions $\mathrm{A} 1, \mathrm{~A} 2$ and $\mathrm{B} 3$, the ratios of neutron fluence measured with TLDs over the one measured with Ta foil are within 0.9 to 1.3. At detector positions A4 (in the maze) and B5 (in the chimney), where lower fluence rates are measured, higher ratios -ranging from 1.6 to 2.3are observed.

Furthermore, the calculations overestimate the measured fluences in all positions except B5 (in the chimney). The discrepancies are mainly attributed to approximations in the geometry model and in particular on the approximations used to describe the instruments and facilities surrounding the tokamak. These components were not possible to be modeled in detail on a realistic timescale, however they attenuate neutrons and therefore calculations over-estimated the neutron fluence in the Hall area. Nevertheless, taking into consideration the overall complexity of the geometry studied, the agreement between calculations and measurements is considered as satisfactory.

\section{Discussion \& Conclusions}

Neutron streaming through tokamak ducts and channels has been studied by several workers [8-15]. Nevertheless, less attention has been given to neutron streaming through larger ducts or labyrinths in the tokamak biological shield. The methodologies generally used for the calculation of neutron streaming along multibent large ducts in accelerator facilities include application of the Monte Carlo technique and the use of predetermined "universal" analytical expressions [16, 17]. The advantage offered by the Monte Carlo simulation approach is the capability to explicitly model the source, geometry and materials configuration encountered.

The similar neutron transmission values obtained in this study for the D-D and the D-T JET plasma discharge sources were attributed to the fact that neutrons have been slowed down due to multiple interactions with the Torus and wall material and their propagation along the labyrinth has become independent of the initial energy of the neutron source and depend only on the labyrinth geometry and material configuration. Elastic neutron scattering in hydrogen and slow neutron absorption in boron in concrete have a significant effect on neutron streaming. Therefore, the results of this study confirmed that the accuracy of the neutron streaming simulations through the JET labyrinth depends on the exact knowledge of the labyrinth geometry and wall composition and is practically independent of the source neutron spectrum.

The calculations were validated by comparison against experimental measurements using both thermoluminescence detectors and activation foils. The results of the comparison showed that in most cases calculations overestimated the measured fluence. Nevertheless, the agreement between experimental results and calculations can be considered as satisfactory taking into consideration the overall complexity of the JET tokamak and shielding configuration studied. Moreover, Vasilopoulou et al [5] studied the effect of concrete composition on neutron streaming calculations along the maze and showed that the accurate knowledge of the hydrogen content in concrete is of outmost importance.

The results of this work contribute to the continuous operational radiation protection effort to minimize collective radiation dose to JET personnel during the current D-D and the forthcoming D-T experimental campaigns. Moreover, they provide important information from JET experience that may assist in the optimization and validation of the radiation shielding design methodology used in ITER.

\section{Acknowledgments}

This work has been started within EFDA and carried out within the framework of the EUROfusion Consortium and has received funding from the European Union's Horizon 2020 research and innovation programme under grant agreement No 633053. The views and opinions expressed herein do not necessarily reflect those of the European Commission.

\section{References}

1. P. Batistoni, S. Conroy, S. Lilley, J. Naish, B. Obryk, S. Popovichev, I.E. Stamatelatos, B.D. Syme, T. Vasilopoulou, Nucl. Fusion 55, 053028 (2015)

2. W.G. McKinney et al., MCNPX overview, Proceedings of the $2006 \mathrm{HSSW}, F N A L, I L, L A-U R-$ 06-6206, Sept. 2006 (2006)

3. A. Santamarina, D. Bernard, P. Blaise, M. Coste, A. Courcelle, T.D. Huynh, C. Jouanne, P. Leconte, O. Litaize, S. Mengelle, G. Noguère, J-M. Ruggiéri, O. Sérot, J. Tommasi, C. Vaglio, J-F. Vidal, The JEFF3.1.1 Nuclear Data Library, JEFF Report 22, NEA No. 6807, OECD, ISBN 978-92-64-99074-6 (2009)

4. D.L. Aldama, A. Trkov, FENDL-2.1: Evaluated nuclear data library for fusion applications, INDC(NDS)-467 (2004)

5. T. Vasilopoulou, I.E. Stamatelatos, P. Batistoni, S. Conroy, B. Obryk, S. Popovichev, B.D. Syme, Radiat. Phys. Chem. 116, 359-364 (2015)

6. International Commission on Radiological Protection (ICRP), The 2007 Recommendations of the International Commission on Radiological Protection, Publication 103, Ann. ICRP 37, 2-4 (2007)

7. B. Obryk, P. Batistoni, S. Conroy, B.D. Syme, S. Popovichev, I.E. Stamatelatos, T. Vasilopoulou, P. Bilski, Fus. Eng. Des. 89, 2235-2240 (2014)

8. M. Angelone, M., Batistoni, P., Petrizzi, L., Pillon, M., Fus. Eng. Des. 51-52, 653-661 (2000)

9. Y. Chen, U. Fischer, S.P. Simakov, F.J. Wasastjern, Nucl. Mater. 367-370, 1580-1585 (2007)

10. S. Sato, K. Maki, Fus. Eng. Des. 65 501-524 (2003)

11. M. Yamauchi, K. Ochiai, Y. Morimoto, M. Wada, S. Sato, T. Nishitani, Radiat. Prot. Dosim. 116, 542-546 (2005) 
12. C. Konno, F. Maekawa, Y. Uno, Y. Kasugai, M. Wada, Y. Ikeda, H. Takeuchi, Fus. Eng. Des. 51-52, 797-802 (2000)

13. K. Ochiai, S. Sato, M. Wada, N. Kubota, K. Kondo, M. Yamauchi, Y. Abe, T. Nishitani, C. Konno, Fus. Eng. Des. 82, 2794-2798 (2007)

14. F. Moro, L. Petrizzi, G. Brolatti, B. Esposito, D. Marocco, R. Villari, Fus. Eng. Des. 84, 1351-1356 (2009)

15. A. Serikov, U. Fischer, D. Leichtle, C.S. Pitcher, Fusion Eng. Des. 87, 690-694 (2012)

16. H. Dinter, D. Dworak, K. Tesch, Nucl. Instr. Meth. Phys. Res. A 333, 507-512 (1993)

17. E. Mauro, M. Silari, Nucl. Instr. Meth. Phys. Res. A, 608, 28-36 (2009) 